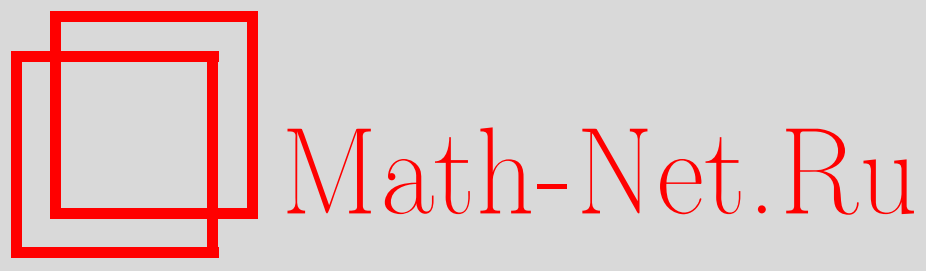

С. В. Асташкин, П. Сунехаг, Вещественный метод интерполяции на парах пересечений, Функи. анализ и его прил., 2006, том 40, выпуск 3, 66-69

DOI: https://doi.org/10.4213/faa744

Использование Общероссийского математического портала Math-Net.Ru подразумевает, что вы прочитали и согласны с пользовательским соглашением

http://www . mathnet.ru/rus/agreement

Параметры загрузки:

IP : 54.198 .55 .26

26 апреля 2023 г., 17:44:25

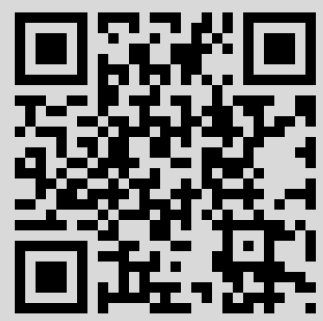




\title{
Вещественный метод интерполяции на парах пересечений
}

\author{
(c) 2006. С. В. АСТАшкин, П. СунехаГ
}

1. Введение. Одной из центральных в теории интерполяции операторов [1] остается проблема интерполяции подпространств, упомянутая еще в монографии Лионса и Мадженеса [2]. Ни в коей мере не претендуя на полноту, упомянем здесь работы [3]-[12], в которых изучались ее различные аспекты. Важным частным случаем этой проблемы является задача интерполяции пересечений, которую для вещественного метода интерполяции (определения см. ниже) можно сформулировать следующим образом. Пусть $\left(X_{0}, X_{1}\right)$ - банахова пара, т.е. два банаховых пространства, линейно и непрерывно вложенных в отделимое линейное топологическое пространство $\mathscr{T}$. Если $N-$ линейное подпространство пространства $\mathscr{T}$, то оно порождает нормированную (вообще говоря, не банахову) пару $\left(X_{0} \cap N, X_{1} \cap N\right)$, где норма в $X_{i} \cap N$ является сужением нормы пространства $X_{i}(i=0,1)$. Спрашивается, при каких условиях на тройку $\left(X_{0}, X_{1}, N\right)$ и параметры вещественного метода интерполяции $\theta \in(0,1)$ и $q \in[1, \infty]$ выполнено следующее естественное равенство (по составу элементов с эквивалентностью норм):

$$
\left(X_{0} \cap N, X_{1} \cap N\right)_{\theta, q}=\left(X_{0}, X_{1}\right)_{\theta, q} \cap N ?
$$

Мы будем рассматривать ситуацию, когда $N$ - ядро линейного функционала $\psi \in\left(X_{0} \cap X_{1}\right)^{*}$. В этом случае равенство (1) означает просто эквивалентность норм пространств $N_{\theta, q}=\left(X_{0} \cap N, X_{1} \cap N\right)_{\theta, q}$ и $X_{\theta, q}=\left(X_{0}, X_{1}\right)_{\theta, q}$ на подпространстве $N$. В работах [13], [14] и независимо в [15] были введены четыре индекса, связанные с функционалом $\psi$ как элементом суммы сопряженных пространств $X_{0}^{*}+X_{1}^{*}$. С их помощью удалось получить ответ при некотором дополнительном условии. В этой заметке мы анонсируем результаты, в которых это условие снято за счет введения еще двух аналогичных индексов. Тем самым здесь получено полное и окончательное решение рассматриваемой задачи.

Важный частный случай описанной задачи был рассмотрен в работе Кругляка, Малигранды и Перссона [16]. При некоторых дополнительных предположениях в ней были найдены условия на $\theta \in(0,1), p \in[1, \infty)$ и весовые функции $w_{0}(x)$ и $w_{1}(x)$, при которых выполнено равенство

$$
\left(L_{p}\left(w_{0}\right) \cap N, L_{p}\left(w_{1}\right) \cap N\right)_{\theta, p}=\left(L_{p}\left(w_{0}\right), L_{p}\left(w_{1}\right)\right)_{\theta, p} \cap N,
$$

где $L_{p}(w)$ - весовое $L_{p}$-пространство на $(0, \infty)$ с обычной нормой, а $N$ - линейное пространство всех функций $f:(0, \infty) \rightarrow \mathbb{R}$, таких, что $\int_{0}^{\infty} f(x) d x=0$. Там же была поставлена аналогичная задача для более общей пары $\left(L_{p_{0}}\left(w_{0}\right), L_{p_{1}}\left(w_{1}\right)\right)$. Используя теоремы 1 и 2 настоящей заметки, нетрудно получить решение этой задачи; в конце работы будет сформулирован один из результатов в этом направлении (подробнее см. [14]).

2. Определения и обозначения. Для нормированной пары $\left(X_{0}, X_{1}\right)$ и $t>0$ определим $\mathscr{K}$-функционал Петре

$$
\mathscr{K}\left(t, x ; X_{0}, X_{1}\right)=\inf _{x=x_{0}+x_{1}, x_{i} \in X_{i}}\left(\left\|x_{0}\right\|_{X_{0}}+t\left\|x_{1}\right\|_{X_{1}}\right), \quad x \in X_{0}+X_{1} .
$$


Если $0<\theta<1$ и $1 \leqslant q<\infty$, то интерполяционное пространство $X_{\theta, q}=$ $\left(X_{0}, X_{1}\right)_{\theta, q}$ вещественного метода состоит из всех $x \in X_{0}+X_{1}$, таких, что

$$
\|x\|_{X_{\theta, q}}=\left\{\int_{0}^{\infty}\left(t^{-\theta} \mathscr{K}\left(t, x: X_{0}, X_{1}\right)\right)^{q} \frac{d t}{t}\right\}^{1 / q}<\infty .
$$

Можно дать и другое определение этого пространства (с эквивалентной нормой), используя $\mathscr{J}$-функционал

$$
\mathscr{J}\left(t, x ; X_{0}, X_{1}\right)=\max \left(\|x\|_{X_{0}}, t\|x\|_{X_{1}}\right), \quad x \in X_{0} \cap X_{1} .
$$

А именно, $X_{\theta, q}$ состоит из всех $x \in X_{0}+X_{1}$, представимых в виде $x=\sum_{k \in \mathbb{Z}} 2^{\theta k} x_{k}$ (сходимость в $\left.X_{0}+X_{1}\right)$, с нормой

$$
\inf \left\{\sum_{k \in \mathbb{Z}}\left(\mathscr{J}\left(2^{k}, x_{k} ; X_{0}, X_{1}\right)\right)^{q}\right\}^{1 / q},
$$

где нижняя грань берется по всевозможным представлениям элемента $x$ указанного вида.

Если $X_{0} \cap X_{1}$ всюду плотно в $X_{0}$ и в $X_{1}$, а $\psi \in\left(X_{0} \cap X_{1}\right)^{*}$, то можно рассмотреть банахову пару сопряженных пространств $\left(X_{0}^{*}, X_{1}^{*}\right)$ и $\psi \in X_{0}^{*}+X_{1}^{*}[1, \S 3.7]$. Важную роль в дальнейшем играет $\mathscr{K}$-функционал $k(t)=\mathscr{K}\left(t, \psi ; X_{0}^{*}, X_{1}^{*}\right)$, а также функции

$$
M(t)=\sup _{s>0} \frac{k(t s)}{k(s)}, \quad M_{0}(t)=\sup _{0<s \leqslant \min (1,1 / t)} \frac{k(t s)}{k(s)}, \quad M_{\infty}(t)=\sup _{s \geqslant \max (1,1 / t)} \frac{k(t s)}{k(s)} .
$$

Они полумультипликативны при $t>0$; поэтому существуют числа

$$
\begin{aligned}
& \alpha=\lim _{t \rightarrow 0} \frac{\log _{2} M(t)}{\log _{2} t}, \quad \alpha_{0}=\lim _{t \rightarrow 0} \frac{\log _{2} M_{0}(t)}{\log _{2} t}, \quad \alpha_{\infty}=\lim _{t \rightarrow 0} \frac{\log _{2} M_{\infty}(t)}{\log _{2} t}, \\
& \beta=\lim _{t \rightarrow \infty} \frac{\log _{2} M(t)}{\log _{2} t}, \quad \beta_{0}=\lim _{t \rightarrow \infty} \frac{\log _{2} M_{0}(t)}{\log _{2} t}, \quad \beta_{\infty}=\lim _{t \rightarrow \infty} \frac{\log _{2} M_{\infty}(t)}{\log _{2} t},
\end{aligned}
$$

называемые индексами растяжения функции $k(t)$. Легко видеть, что $0 \leqslant \alpha \leqslant$ $\alpha_{0} \leqslant \beta_{0} \leqslant \beta \leqslant 1$ и $0 \leqslant \alpha \leqslant \alpha_{\infty} \leqslant \beta_{\infty} \leqslant \beta \leqslant 1$.

3. Основные результаты. Предположим, что $\left(X_{0}, X_{1}\right)$ - банахова пара, такая, что $X_{0} \cap X_{1}$ всюду плотно в $X_{0}$ и в $X_{1}$. Пусть $\psi \in\left(X_{0} \cap X_{1}\right)^{*}, \psi \neq 0$, $N=\operatorname{Ker} \psi$ и $N_{i}$ - пространство $N$, рассматриваемое с нормой пространства $X_{i}(i=0,1)$.

Теорема 1. Нормы интерполяционных пространств $N_{\theta, q}=\left(N_{0}, N_{1}\right)_{\theta, q} u$ $X_{\theta, q}=\left(X_{0}, X_{1}\right)_{\theta, q}$ эквивалентны на $N$ тогда и только тогда, когда

$$
\theta \in(0, \alpha) \cup\left(\beta_{\infty}, \alpha_{0}\right) \cup\left(\beta_{0}, \alpha_{\infty}\right) \cup(\beta, 1) .
$$

$\Pi$ пи этом, если $\theta \in(0, \alpha) \cup\left(\beta_{0}, \alpha_{\infty}\right) \cup(\beta, 1)$, то $N_{\theta, q}=\left(N_{0}, N_{1}\right)_{\theta, q}$ всюду плотно в $X_{\theta, q}=\left(X_{0}, X_{1}\right)_{\theta, q}$, а если $\theta \in\left(\beta_{\infty}, \alpha_{0}\right)$, то $N_{\theta, q}$ всюду плотно в некотором подпространстве коразмерности 1 пространства $X_{\theta, q}$.

ЗАмЕчАНиЕ 1 . Ввиду неравенств $\alpha_{0} \leqslant \beta_{0}$ и $\alpha_{\infty} \leqslant \beta_{\infty}$ непустым может быть, самое большее, лишь один из интервалов $\left(\beta_{0}, \alpha_{\infty}\right)$ и $\left(\beta_{\infty}, \alpha_{0}\right)$. 
Иную формулировку теоремы 1 можно дать, используя следующее определение из [15]. Сохраняя прежние обозначения и условия, определим $X_{\theta, q, \psi}$ как множество всех $x \in X_{0}+X_{1}$, допускающих представление вида

$$
x=\sum_{k \in \mathbb{Z}} 2^{\theta k} x_{k}, \quad x_{k} \in N\left(\text { сходимость в } X_{0}+X_{1}\right),
$$

с нормой (3), где нижняя грань берется по представлениям вида (5).

Теорема 2. Для того чтобъ пространство $X_{\theta, q, \psi}$ было замкнуто в пространстве $X_{\theta, q}=\left(X_{0}, X_{1}\right)_{\theta, q}$, необходимо и достаточно выполнение условия (4).

При этом, если $\theta \in(0, \alpha) \cup\left(\beta_{0}, \alpha_{\infty}\right) \cup(\beta, 1)$, mо $X_{\theta, q, \psi}=X_{\theta, q}$, а если $\theta \in\left(\beta_{\infty}, \alpha_{0}\right)$, mо $X_{\theta, q, \psi}=X_{\theta, q} \cap \operatorname{Ker} \widetilde{\psi}$, где $\widetilde{\psi}-$ непрерьвное продолжение бункиионала $\psi$ на пространство $X_{\theta, q}$.

ЗАмечАниЕ 2. Теорема 2 является усилением предложения 5.6 из работы [15], где дополнительно предполагалось, что $\beta_{\infty} \leqslant \alpha_{0}$.

ЗАмечАние 3. Так же, как и в [13]-[15], в доказательстве мы используем идею сведения задачи интерполяции пересечений к изучению оператора сдвига в некотором весовом $l_{q}$-пространстве последовательностей. Впервые она была применена Ивановым и Калтоном [10] для сравнения интерполяционных пространств $\left(X_{0}, X_{1}\right)_{\theta, q}$ и $\left(N_{0}, X_{1}\right)_{\theta, q}$, где $\psi \in X_{0}^{*}$ и $N_{0}=\operatorname{Ker} \psi$. Этот случай является частным по отношению к рассматриваемому здесь, и поэтому интерполяционные результаты из [10] являются следствием теоремы 1 настоящей заметки (см. [14] и [15]).

4. Об интерполяции пересечений весовых $L_{p}$-пространств, порожденных интегральным функционалом. Пусть $w(x)$ - положительная измеримая на $(0, \infty)$ функция, $1 \leqslant p \leqslant \infty$. Тогда, как обычно, $L_{p}(w)$ - пространство всех измеримых функций $f:(0, \infty) \rightarrow \mathbb{R}$ с нормой $\|f\|_{w, p}=\left(\int_{0}^{\infty}|f(x)|^{p} w(x) d x\right)^{1 / p}$ (с естественной модификацией при $p=\infty$ ). Рассмотрим линейный функционал $\varphi(f)=\int_{0}^{\infty} f(x) d x$ с областью определения, состоящей из всех измеримых функций $f:(0, \infty) \rightarrow \mathbb{R}$, таких, что $\int_{a}^{b}|f(x)| d x<\infty$ для произвольных $0<a<b<\infty$ и существует $\lim _{a \rightarrow 0, b \rightarrow \infty} \int_{a}^{b} f(x) d x$. Обозначим его ядро через $N$.

Предположим, что сужение функционала $\varphi$ на пересечение $L_{p_{0}}\left(w_{0}\right) \cap L_{p_{1}}\left(w_{1}\right)$ ограничено. Тогда, основываясь на теореме 1 и действуя так же, как в [14], мы можем найти необходимые и достаточные условия, при которых выполнен аналог равенства $(2)$ для пары $\left(L_{p_{0}}\left(w_{0}\right), L_{p_{1}}\left(w_{1}\right)\right)$ (уже без всяких ограничений на индексы растяжения $\mathscr{K}$-функционала Петре $\varphi$ в паре $\left.\left(L_{p_{0}}\left(w_{0}\right)^{*}, L_{p_{1}}\left(w_{1}\right)^{*}\right)\right)$. Не вдаваясь в подробности, приведем здесь лишь один наиболее просто формулируемый результат в случае $p_{0}=p_{1}=1$. Пусть $w(x)=w_{0}(x) / w_{1}(x)$, а $w^{-1}(x)-$ функция, обратная к функции $w(x)$.

TEOPEMA 3. Пусть $w_{0}(x)$ возрастает, $w_{1}(x)$ yбъвает $u \lim _{x \rightarrow 0} w(x)=$ $\lim _{x \rightarrow \infty} 1 / w(x)=0$. Если $\alpha, \beta, \alpha_{0}, \beta_{0}, \alpha_{\infty} u \beta_{\infty}-$ индексы растяжения функции $k(t)=1 /\left(w_{0}\left(w^{-1}(1 / t)\right)\right)$, то равенство

$$
\left(L_{1}\left(w_{0}\right) \cap N, L_{1}\left(w_{1}\right) \cap N\right)_{\theta, q}=\left(L_{1}\left(w_{0}\right), L_{1}\left(w_{1}\right)\right)_{\theta, q} \cap N
$$

верно тогда и только тогда, когда выполнено (4). 


\section{ЛитерАтУра}

[1] Й. Берг, Й. Лефстрем, Интерполяиионные пространства. Введение, Мир, М., 1980. [2] Ж. Л. Лионс, Э. Мадженес, Неоднородные граничные задачи и их приложения, Мир, М., 1971. [3] H. Triebel, Math. Nachr., 69 (1975), 57-60. [4] L. Maligranda, Suppl. Rend. Circ. Mat. Palermo, 10 (1985), 113-118. [5] R. Wallsten, in: Lecture Notes in Math., vol. 1902, 1988, 410-419. [6] G. Pisier, Pacific J. Math., 155 (1992), 341368. [7] S. Janson, Arkiv Math., 31 (1993), 307-338. [8] С. В. Кисляков, Куанхуа Шу, Алгебра и анализ, 8:4 (1996), 75-109. [9] J. Löfström, Interpolation of subspaces, Technical report no. 10, Univ. of Göteborg, 1997. [10] S. Ivanov, N. Kalton, Алгебра u анализ, 13:2 (2001), 93-115. [11] S. V. Astashkin, J. Math. Math. Sci., 25:7 (2001), 451465. [12] S. Kaijser, P. Sunehag, in: Function Spaces, Interpolation Theory and Related Topics (Lund 2000), de Gruyter, Berlin, 2002, 345-353. [13] C. В. Асташкин, Функu. анализ и его прил., 39:2 (2005), 61-64. [14] С. В. Асташкин, Алгебра и анализ, 17:2 (2005), 33-69. [15] P. Sunehag, J. Approx. Theory, 130 (2004), 78-98. [16] N. Krugljak, L. Maligranda, L.-E. Persson, Arkiv Mat., 37 (1999), 323-344.

Самарский госуниверситет

e-mail: astashkn@ssu.samara.ru

Поступило в редакцию 20 апреля 2005 г.

Department of Mathematics, Uppsala University

e-mail: peter@math.uu.se

УДК 517.986.4

\section{Об операторах Рака*}

(C) 2006. Р. С. ИСмАГИлов

Обычно коэффициенты Рака́ определяются для тензорных произведений конечномерных представлений полупростых групп Ли ([1], [2]). В дальнейшем это определение переносилось на представления со старшим весом группы $S L(2, \mathbb{R})$; см. [3]. В этом случае спектр произведения дискретен, что позволяет определить указанные коэффициенты «аналитическим продолжением по целочисленным параметрам» из формул для конечномерного случая. В работе [5] указаны коэффициенты Рака для трех представлений этой же группы в предположении, что хотя бы два из них содержатся в дискретной серии.

В этой заметке в качестве обобщения коэффициентов Рака вводятся операторы Рака для произвольной локально компактной группы с «хорошим» двойственным пространством. Приводятся явные формулы для операторов Рака, связанных с представлениями основной серии группы $G=P S L(2, \mathbb{C})$ (тензорные произведения этих представлений были изучены Наймарком [4]).

1. Операторы Рака. Введем основное определение.

Пусть $G$ - локально компактная группа типа I и $\widehat{G}$ - двойственное пространство. Пусть дано непрерывное поле гильбертовых пространств $H_{c}$ и действующих в них представлений $T_{c}\left(T_{c}\right.$ принадлежит классу эквивалентности $c \in \widehat{G})$. Через $\delta c$ обозначим меру Планшереля на $\widehat{G}$.

*Работа выполнена при поддержке РФФИ, грант 05-01-0001. 